 \\ MOUNTaiNS \\ Mathematical Publications
}

\section{ON STRONGLY COUNTABLY CONTINUOUS FUNCTIONS}

\author{
Grażyna HorbaCZEWSKA
}

\begin{abstract}
A real-valued function $f$ on $\mathbb{R}$ is strongly countably continuous provided that there is a sequence of continuous functions $\left(f_{n}\right)_{n \in \mathbb{N}}$ such that the graph of $f$ is contained in the union of the graphs of $f_{n}$.

Some examples of interesting strongly countably continuous functions are given: one for which the inverse function is not strongly countably continuous, another which is an additive discontinuous function with a big image and a function which is approximately and $I$-approximately continuous, but it is not strongly countably continuous.
\end{abstract}

Definition 1. A function $f: \mathbb{R} \rightarrow \mathbb{R}$ is countably continuous if there exists a family of sets $\left\{A_{n}\right\}_{n=1}^{\infty}$ such that $\bigcup_{n=1}^{\infty} A_{n}=\mathbb{R}$ and $f \mid A_{n}$, the restriction of $f$ to $A_{n}$ is continuous.

Definition 2 (GFG]). A function $f: \mathbb{R} \rightarrow \mathbb{R}$ is strongly countably continuous if there is a sequence of continuous functions $f_{n}: \mathbb{R} \rightarrow \mathbb{R}$ such that $f \subset \bigcup_{n=1}^{\infty} f_{n}$.

Of course, if $g$ is strongly countably continuous, then it is countably continuous (because $\mathbb{R}=\bigcup_{n=1}^{\infty} A_{n}$, where $A_{n}=\left\{x \in \mathbb{R}: f(x)=f_{n}(x)\right\}$ and $f\left|A_{n}=f_{n}\right| A_{n}$ is continuous for $n \in \mathbb{N}$ ).

But, the class of strongly countably continuous functions is essentially weaker than the class of the countably continuous functions. For example, each monotone function is countably continuous since it has a countable set of points of discontinuity, but if this set is dense, then the function cannot be strongly countably continuous ( $\mathrm{GFG}, \mathrm{CL}$ ).

For basic properties of the class of countably continuous functions, we refer the reader to $\mathrm{GFG}$.

THEOREM 1. If functions $f, g: \mathbb{R} \rightarrow \mathbb{R}$ are strongly countably continuous and $F: \mathbb{R}^{2} \rightarrow \mathbb{R}$ is also strongly countably continuous, then the function $x \rightarrow F(f(x)$, $g(x))$ is also strongly countably continuous.

2000 Mathematics Subject Classification: 26A15, 26A21.

Keywords: countable decomposition, additive functions, approximate and $I$-approximate continuity, Baire*1 functions. 
We omit the definition of a strong countable continuity for the function defined on the plane but it is an obvious generalization.

P r o of. By assumption there exist sequences of continuous functions $\left\{f_{n}\right\}_{n \in \mathbb{N}}$, $\left\{g_{n}\right\}_{n \in \mathbb{N}},\left\{F_{n}\right\}_{n \in \mathbb{N}}$ such that

$$
f_{n}, g_{n}: \mathbb{R} \rightarrow \mathbb{R}, \quad F_{n}: \mathbb{R}^{2} \rightarrow \mathbb{R}, \quad n \in \mathbb{N}
$$

and

$$
f \subset \bigcup_{n \in \mathbb{N}} f_{n}, \quad g_{n} \subset \bigcup_{n \in \mathbb{N}} g_{n}, \quad F \subset \bigcup_{n \in \mathbb{N}} F_{n} .
$$

Let

$$
\begin{aligned}
& A_{n}=\left\{x \in \mathbb{R}: f_{n}(x)=f(x)\right\}, \\
& B_{n}=\left\{x \in \mathbb{R}: g_{n}(x)=g(x)\right\}, \\
& Z_{n}=\left\{(x, y) \in \mathbb{R}^{2}: F_{n}(x)=F(x)\right\}, \quad n \in \mathbb{N} .
\end{aligned}
$$

Then

$$
\bigcup_{n \in \mathbb{N}} A_{n}=\bigcup_{n \in \mathbb{N}} B_{n}=\mathbb{R} \quad \text { and } \bigcup_{n \in \mathbb{N}} Z_{n}=\mathbb{R}^{2} .
$$

Let $G: \mathbb{R} \rightarrow \mathbb{R}^{2}$ be a function given by the following formula:

$$
G(x)=(f(x), g(x)), \quad x \in \mathbb{R},
$$

and let

$$
C_{n, k, i}=A_{n} \cap B_{k} \cap G^{-1}\left(Z_{i}\right), \quad n, k, i \in \mathbb{N} .
$$

Obviously,

$$
\bigcup_{n, k, i \in \mathbb{N}} C_{n, k, i}=\mathbb{R}
$$

and if $C_{n, k, i} \neq \emptyset$, then for $x \in C_{n, k, i}$ we have $f(x)=f_{n}(x), g(x)=g_{k}(x)$ and $(f(x), g(x)) \in Z_{i}$ so,

$$
F(f(x), g(x))=F_{i}(f(x), g(x))=F_{i}\left(f_{n}(x), g_{k}(x)\right) .
$$

Therefore, the function $x \rightarrow F(f(x), g(x))$ is continuous on $C_{n, k, i}, n, k, i \in \mathbb{N}$ and $F(f, g) \subset \bigcup_{n, k, i \in \mathbb{N}} F_{i}\left(f_{n}, g_{n}\right)$.

The last theorem is slightly stronger than an analogous one in GFG], but the following corollary comes from [GFG].

CorollaRY 1. If $a, b \in \mathbb{R}$ are constants, $f, g: \mathbb{R} \rightarrow \mathbb{R}$ are strongly countably continuous functions then the functions af $+b g, f \cdot g, \max (f, g), \min (f, g)$ and $f \circ g$ are also strongly countably continuous. If $g(x) \neq 0$ for all $x \in \mathbb{R}$, then the quotient $f \mid g$ is also strongly countably continuous. 


\section{ON STRONGLY COUNTABLY CONTINUOUS FUNCTIONS}

EXAMPLE 1. There is a strongly countably continuous function for which the inverse function is not strongly countably continuous.

Let $g:[0,1] \rightarrow[0,1]$ be an increasing, left-hand continuous function satisfying the condition $g(1)=1$ and $g(0)=0$, with a countable dense set of points of discontinuity denoted by $D=\left\{y_{1}, y_{2}, \ldots\right\}$.

Let $\left\{I_{n}\right\}_{n \in \mathbb{N}}$ be a sequence of intervals, where $I_{n}=\left(g\left(y_{n}\right), \lim _{y \rightarrow y_{n}^{+}} g(y)\right)$ for $n \in \mathbb{N}$. We see, at once, that $I_{i} \cap I_{j}=\emptyset$ for $i \neq j$ and $g([0,1])=[0,1] \backslash \bigcup_{n=1}^{\infty} I_{n}$.

Let $f:[0,1] \rightarrow\left[0,1+\sum_{n=1}^{\infty} \lambda\left(I_{n}\right)\right]$ be defined by a formula:

$$
f(x)= \begin{cases}g^{-1}(x) & \text { for } x \in[0,1] \backslash \bigcup_{n=1}^{\infty} I_{n}, \\ 1+\sum_{i=1}^{n-1} \lambda\left(I_{i}\right)+\left(x-\inf I_{n}\right) & \text { for } x \in I_{n}, n \in \mathbb{N},\end{cases}
$$

where $\lambda(I)$ denotes the length of an interval $I$.

It is easy to check out that $f$ is strongly countably continuous. We have to construct a sequence of continuous functions such that $f \subset \bigcup_{n=0}^{\infty} f_{n}$. Let $f_{0}$ be an extension of the function $f$ from $[0,1] \backslash \bigcup_{n=1}^{\infty} I_{n}$ to $[0,1]$ given by the formula

$$
f_{0}(x)= \begin{cases}g^{-1}(x) & \text { for } x \in[0,1] \backslash \bigcup_{n=1}^{\infty} I_{n}, \\ y_{n} & \text { for } x \in I_{n}, n \in \mathbb{N}\end{cases}
$$

and for each $n \in \mathbb{N}$, let $f_{n}$ be an extension of the function $f$ from $I_{n}$ to $[0,1]$ given by the formula

$$
f_{n}(x)=1+\sum_{i=1}^{n-1} \lambda\left(I_{n}\right)+\left(x-\inf I_{n}\right) \quad \text { for } \quad x \in[0,1] .
$$

This sequence fulfils the desired conditions, so $f$ is strongly countably continuous.

Suppose, contrary to our claim, that the inverse function $f^{-1}$ is strongly countably continuous. Since $g \subset f^{-1}$, it follows that the function $g=f_{\mid[0,1]}^{-1}$ should be also strongly countably continuous, which is not the case (see GFG, Example 1]).

Now, we want to look at our class of functions in the context of additive functions.

Definition $3(\underline{\mathrm{K}})$. A function $f: \mathbb{R} \rightarrow \mathbb{R}$ is additive if it satisfies Cauchy's equation

$$
f(x+y)=f(x)+f(y) \quad \text { for all } \quad x, y \in \mathbb{R} .
$$

We wish to investigate how large an image for a strongly countably continuous additive but discontinuous function can be. We know that an image for such a function can be uncountable ( $\mathrm{GFG}$ ). 
EXAMPLE 2. There is a strongly countably continuous additive discontinuous function with an image which intersects every uncountable Borel subset of $\mathbb{R}$. Consequently, its image is a set of the full outer measures and of the second category everywhere.

Let $H \subset \mathbb{R}$ be a Burstin basis, it means, a Hamel basis which intersects every uncountable Borel subset of $\mathbb{R}$ (such a basis exists - see [K]). The construction of the desired function appears in GFG].

Fix a point $h_{0} \in H$ and let

$$
f(x)= \begin{cases}x & \text { for } x \in H \backslash\left\{h_{0}\right\}, \\ 0 & \text { for } x=h_{0} .\end{cases}
$$

Then $f(H)=\left(H \backslash\left\{h_{0}\right\}\right) \cup\{0\}$. Since $\frac{f(x)}{x}$ is not a constant function, the additive extension $g: \mathbb{R} \rightarrow \mathbb{R}$ of $f: H \rightarrow \mathbb{R}$ is discontinuous (see [K]). The function $g$ is strongly countably continuous (see [GFG] for more details). The image $g(\mathbb{R})$ contains $H \backslash\left\{h_{0}\right\}$. It is easy to check out that if a set intersects every uncountable Borel subset of $\mathbb{R}$, then this intersection contains at least two points, so the image $g(\mathbb{R})$ is as we want it to be.

Suppose, the set $g(\mathbb{R})$ is not of the full outer measure. Then its complement has a positive inner measure, hence it contains a $F_{\sigma}$ set with a positive measure (an uncountable Borel set), a contradiction. Similarly, if there exists an interval $I$, such that $I \backslash g(\mathbb{R})$ is residual on $I$, then it contains a $G_{\delta}$ set of the second category (an uncountable Borel set), which is impossible.

In [GFG], we can find an example of a bounded approximately continuous function which is not strongly countably continuous.

EXAMPLE 3. There exists a bounded function which is approximately and $I$ -approximately continuous but it is not strongly countably continuous.

For relevant definitions, look in CLO

We use here a function from [CLO, p. 84].

Let $\left\{q_{n}\right\}_{n \in \mathbb{N}}$ be a sequence of all rational numbers and $E=\bigcup_{n \in \mathbb{N}}\left[a_{n}, b_{n}\right]$ be an interval set for which 0 is a right-side dispersion and $I$-dispersion point.

Put

$$
f(x)=\left\{\begin{array}{lr}
0 & \text { for } x \notin E, \\
\frac{\operatorname{dist}\left(\{x\},\left(a_{n}, b_{n}\right)^{c}\right)}{b_{n}-a_{n}}, & x \in\left[a_{n}, b_{n}\right]
\end{array}\right.
$$

and let $h_{n}(x)=\frac{2}{3^{n}} f\left(x-q_{n}\right)$ for $n \in \mathbb{N}$.

Then $h_{n}(x) \in\left[0, \frac{1}{3^{n}}\right]$ for $x \in \mathbb{R}, n \in \mathbb{N}$ and $\operatorname{osc}\left(h_{n}, q_{n}\right)=\frac{1}{3^{n}}$. We define $g_{n}=\sum_{i \leq n} h_{i}$ for $n \in \mathbb{N}$ and $g=\lim _{n \rightarrow \infty} g_{n}$. Since $\left|g(x)-g_{n}(x)\right|<\frac{1}{3^{n+1}}$ for $x \in \mathbb{R}, n \in \mathbb{N}$, the sequence converges uniformly. 


\section{ON STRONGLY COUNTABLY CONTINUOUS FUNCTIONS}

Each function $h_{n}$ is approximately and $I$-approximately continuous since it is a linear function on intervals except the point $q_{n}$ and it is a constant function on the neighbourhood of $q_{n}$ in density and $I$-density topology. Since the families of approximately and $I$-approximately continuous functions are closed under addition and uniform convergence, the function $g$ is also approximately and $I$ approximately continuous function.

Assume that $g$ is strongly countably continuous, i.e, there exists a sequence of continuous functions $f_{n}: \mathbb{R} \rightarrow \mathbb{R}, n \in \mathbb{N}$ such that $g \subset \bigcup_{n=1}^{\infty} f_{n}$.

Let $A_{n}=\left\{x: f(x)=f_{n}(x)\right\}$ for $n \in \mathbb{N}$. Then $\mathbb{R}=\bigcup_{n=1}^{\infty} A_{n}$, therefore there exist $n_{0} \in \mathbb{N}$ and an open interval $J$ such that $J \subset \overline{A_{n_{0}}}$. Define $k_{o}=\min \{k \in$ $\left.\mathbb{N}: q_{k} \in J\right\}$. Since $\operatorname{osc}\left(g, q_{k_{0}}\right)=\frac{1}{3^{k_{0}}}$, and $g$ is not continuous from the right-side (see [CLO, p. 83]), so there exist two decreasing sequences $\left\{x_{i}\right\}_{i \in \mathbb{N}}$ and $\left\{z_{i}\right\}_{i \in \mathbb{N}}$ of points from $J$ tending to $q_{k_{0}}$ such that

$$
\left|\lim _{i \rightarrow \infty} g\left(x_{i}\right)-\lim _{i \rightarrow \infty} g\left(z_{i}\right)\right|=\frac{1}{3^{k_{0}}} .
$$

Fix $i \in \mathbb{N}$. If $x_{i} \notin Q$, then $\operatorname{osc}\left(g, x_{i}\right)=0$. If $x_{i} \in Q$, then there exists an index $k>k_{0}$ such that $x_{i}=q_{k}$, hence $\operatorname{osc}\left(g, x_{i}\right)=\frac{1}{3^{k}} \leq \frac{1}{3} \cdot \operatorname{osc}\left(g, q_{k_{0}}\right)=\frac{1}{3} \cdot \frac{1}{3^{k_{0}}}$. In both cases, $\operatorname{osc}\left(g, x_{i}\right) \leq \frac{1}{3^{k}} \leq \frac{1}{3} \operatorname{osc}\left(g, q_{k_{0}}\right)$. Similarly, we obtain $\operatorname{osc}\left(g, z_{i}\right) \leq$ $\frac{1}{3} \operatorname{osc}\left(g, q_{k_{0}}\right)$.

Since $A_{n_{0}}$ is dense on $J$, then for every $i \in \mathbb{N}$ there exists a point $\hat{x}_{i} \in J \cap A_{n_{0}}$ such that

$$
\left|\hat{x}_{i}-x_{i}\right|<\frac{1}{i} \text { and }\left|g\left(x_{i}\right)-g\left(\hat{x}_{i}\right)\right|<\frac{3}{7} \operatorname{osc}\left(g, q_{k_{0}}\right)
$$

and there exists a point $\hat{z}_{i} \in J \cap A_{n_{0}}$ such that

$$
\left|\hat{z}_{i}-z_{i}\right|<\frac{1}{i} \text { and }\left|g\left(z_{i}\right)-g\left(\hat{z}_{i}\right)\right|<\frac{3}{7} \operatorname{osc}\left(g, q_{k_{0}}\right) .
$$

Obviously,

$$
\lim _{i \rightarrow \infty} \hat{x}_{i}=q_{k_{0}} \quad \text { and } \quad \lim _{i \rightarrow \infty} \hat{z}_{i}=q_{k_{0}}
$$

and

$$
\liminf _{i \rightarrow \infty}\left|g\left(\hat{x}_{i}\right)-g\left(\hat{z}_{i}\right)\right| \geq\left(1-\frac{3}{7}-\frac{3}{7}\right) \operatorname{osc}\left(g, q_{k_{0}}\right)=\frac{1}{7} \operatorname{osc}\left(g, q_{k_{0}}\right),
$$

but simultaneously,

$$
g\left(\hat{x}_{i}\right)=f_{n_{0}}\left(\hat{x}_{i}\right) \text { and } g\left(\hat{z}_{i}\right)=f_{n_{0}}\left(\hat{z}_{i}\right)
$$

since $\hat{x}_{i}, \hat{z}_{i} \in A_{n_{0}}$.

Therefore, the function $f_{n_{0}}$ is not continuous at the point $q_{k_{0}}$ - a contradiction.

Remark 1. The function presented above is countably continuous since its set of discontinuity is countable. 


\section{GRAŻYNA HORBACZEWSKA}

This function is of the first Baire class but not Baire* 1 , since to be Baire* 1 , a function should be continuous on a dense open set.

The following characterization of Baire* 1 function was given by O' Malley:

Theorem 2 (['] $]$ ). A function $f:[0,1] \rightarrow \mathbb{R}$ is Baire ${ }^{*} 1$ if and only if there is a sequence of closed sets $E_{n}$ such that $\bigcup_{n \in \mathbb{N}} E_{n}=[0,1]$ and $f \mid E_{n}$ is continuous for each $n \in \mathbb{N}$.

\section{REFERENCES}

[CL] CSASZAR, A.-LACZKOVICH, M.: Some remarks on discrete Baire classes, Acta Math. Acad. Sci. Hungar. 33 (1979), 51-70.

[CLO] CIESIELSKI, K.-LARSON, L.-OSTASZEWSKI, K.: I-density Continuous Functions, Mem. Amer. Math. Sci., Vol. 107, AMS, Providence, 1994.

[GFG] GRANDE, Z.-FATZ-GRUPKA, A.: On countably continuous functions, Tatra Mt. Math. Publ. 28 (2004) 57-63.

[K] KUCZMA, M.: An Introduction to the Theory of Functional Equations and Inequalities. PWN, Warszawa, 1985.

[O'M] O'MALLEY, R. J.: Approximately differentiable functions: The r-topology, Pacific J. Math. 72 (1977), 207-222.

Department of Mathematics and Computer Science

Łódź University

Banacha 22

PL-90-238 Eódź

POLAND

E-mail: grhorb@math.uni.lodz.pl 